\title{
Facile One-Pot Multicomponent Synthesis of Biaryl Tetrazoles through In Situ Formed Secondary Amides
}

\author{
K. VIJAYAKUMAR and B. R. VENKATRAMAN* \\ Post Graduate and Research Department of Chemistry, \\ Periyar E. V. R. College (Autonomous), Tiruchirappalli. Tamilnadu, India \\ brvenkatraman@yahoo.com
}

Received 13 July 2017 / Accepted 20 July 2017

\begin{abstract}
A series of novel tetrazoles was synthesized through the multicomponent reactions of piperonal, aryl diamines and aroyl chlorides in a one-pot fashion. All the synthesized products were characterized by elemental analyses, FT-IR, ${ }^{1} \mathrm{H}$ NMR ${ }^{13} \mathrm{C}$ NMR and LC-Mass spectral data.
\end{abstract}

Keywords: Tetrazole, Piperonal, Aryldiamines

\section{Introduction}

Tetrazoles are synthetic compounds with the highest nitrogen contents among the stable heterocycles ${ }^{1}$. They have long been recognized as carboxylic acid isostere ${ }^{2,3}$ and are important heterocycles in medicinal chemistry, owing to their increased stability towards metabolic degradation pathways ${ }^{4}$. The acidity of the tetrazole $\mathrm{NH}$ group corresponds roughly to that of the carboxylic acid ${ }^{5}$. Interest in tetrazole chemistry over the past few years has been increasing rapidly, mainly as a result of the role played by this heterocyclic functionality in medicinal chemistry as a metabolically stable surrogate for carboxylic acid functionalities ${ }^{6-8}$. Tetrazoles are well known compounds with a number of biological activities ${ }^{9-12}$ such as antibacterial, antifungal and analgesic. Piperonal is a molecule widely used as basis for the heliotrope-type perfumes and for cosmetic preparations, being also applied as an intermediate for agrochemical and pharmaceutical products. It has been moreover demonstrated that piperonal has powerful aroma therapeutic qualities able to elevate mood and to impart a general wellbeing $^{13-17}$.

In addition, synthetic compounds containing piperonyl ring possess enormous biological activity such as anti-cancer ${ }^{18,19}$, anti-convulsant ${ }^{20,21}$, anti-amoebic ${ }^{22}$, antiproliferative $^{23}$, antiviral ${ }^{24}$, anti-tumor ${ }^{25}$, anti-plasmodial ${ }^{26}$, COX-2 inhibitor ${ }^{27}$. Considering the importance of tetrazole and piperonyl molecules in pharmaceutical filed, we have developed a series of piperonyl-tetrazolo compounds. 


\section{Experimental}

All the reagents were purchased from Sigma-Aldrich. Solvents were purchased from Finar chemicals and purified prior to use. The reactions were monitored by analytical TLC on silica gel G/GF 254 plates and column chromatography was performed with 60-120 mesh silica gel. Melting points were determined on a veego (India) capillary melting point apparatus and are uncorrected. Infrared spectra were recorded on a Perkin-Elmer FT-IR spectrometer by using $\mathrm{KBr} .{ }^{1} \mathrm{H}$ and ${ }^{13} \mathrm{C}$ NMR spectra were recorded in Bruker 300 and $75 \mathrm{MHz}$ spectrometer.

\section{General procedure for the synthesis of compounds (4a-4o)}

To a stirred solution of benzo[1,3]dioxole-5-carbaldehyde 1a $(0.005 \mathrm{~mol})$, phenyldiamine 2a-2c $(0.005 \mathrm{~mol})$ and benzoyl chlorides 3a-3e $(0.005 \mathrm{~mol})$ were added and refluxed for $4 \mathrm{~h}$ at $70{ }^{\circ} \mathrm{C}$. To this reaction mixture $\mathrm{KOH}(1 \mathrm{mmol}), \mathrm{PCl}_{5}(0.001 \mathrm{~mol})$ and $\mathrm{NaN}_{3}(0.005 \mathrm{~mol})$ were added and stirred for $6 \mathrm{~h}$ at $80^{\circ} \mathrm{C}$. The reaction progress was monitored by TLC by using ethyl acetate-hexane (80:20\%). After the completion of the reaction, the solvent was evaporated in vacuo and the residue was diluted with $\mathrm{CH}_{2} \mathrm{Cl}_{2}(50 \mathrm{~mL})$ and washed with saturated $\mathrm{NH}_{4} \mathrm{Cl}$ $(20 \mathrm{~mL})$ and water $(20 \mathrm{~mL})$. Drying $\left(\mathrm{Na}_{2} \mathrm{SO}_{4}\right)$ and evaporation of the solvent gave a residue that was purified on silica gel column chromatography using $n$-hexane/ethyl acetate as eluent.

4(E)-N-(benzo[d][1,3]dioxol-5-ylmethylene)-2-(5-phenyl-1H-tetrazol-1-yl)aniline (4a)

Anal. Calcd. (\%) for $\mathrm{C}_{21} \mathrm{H}_{15} \mathrm{~N}_{5} \mathrm{O}_{2}$ : C, 68.28; H, 4.09; N, 18.96. Found (\%): C, 68.26; H, $4.08 ; \mathrm{N}$, 18.94; Pale yellow solid; m. p. $166-168^{\circ} \mathrm{C} ; \mathrm{R}_{\mathrm{f}}=0.41$; FT-IR $\left(\mathrm{KBr}, \mathrm{cm}^{-1}\right): 1609,1586 ;{ }^{1} \mathrm{H}$ NMR (300 MHz, $\left.\mathrm{CDCl}_{3}, \delta / \mathrm{ppm}\right): 9.22(\mathrm{~s}, 1 \mathrm{H}), 7.77-6.35(\mathrm{~m}, 12 \mathrm{H}), 6.02(\mathrm{~s}, 2 \mathrm{H}) ;{ }^{13} \mathrm{C}$ NMR $(75 \mathrm{MHz}$, DMSO- $\left.d_{6}, \delta / \mathrm{ppm}\right): 165.49,158.01,150.66,147.28,145.48,138.50,134.11,132.59,130.26$, 129.05, 128.51, 128.12, 126.51, 120.28, 108.17, 106.56, 101.73; LC-MS (m/z): 369.1249.

(E)-N-(benzo[d][1,3]dioxol-5-ylmethylene)-2-(5-(4-chlorophenyl)-1H-tetrazol-1yl)aniline $(\mathbf{4 b})$

Anal. Calcd. (\%) for $\mathrm{C}_{21} \mathrm{H}_{14} \mathrm{ClN}_{5} \mathrm{O}_{2}$ : C, 62.46; H, 3.49 ; N, 17.34. Found (\%): C, 68.44; H, 3.46; $\mathrm{N}, 17.31$. Yellow solid; m. p.180-182 ${ }^{\circ} \mathrm{C} ; \mathrm{R}_{\mathrm{f}}=0.47$. FT-IR $\left(\mathrm{KBr}, \mathrm{cm}^{-1}\right): 1652,1596 ;{ }^{1} \mathrm{H}$ NMR (300 MHz, $\left.\mathrm{CDCl}_{3}, \delta / \mathrm{ppm}\right): 9.76(\mathrm{~s}, 1 \mathrm{H}), 7.97-6.50(\mathrm{~m}, 11 \mathrm{H}), 5.98(\mathrm{~s}, 2 \mathrm{H}) ;{ }^{13} \mathrm{C} \mathrm{NMR}(75 \mathrm{MHz}$, DMSO- $\left.d_{6}, \delta / \mathrm{ppm}\right): 165.13,156.58,150.81,147.58,145.05,137.52,134.90,131.52,130.73$, 128.51, 127.82, 125.43, 121.55, 120.86, 108.25, 106.19, 101.58; LC-MS (m/z): 403.1114.

(E)-N-(benzo[d][1,3]dioxol-5-ylmethylene)-2-(5-(4-fluorophenyl)-1H-tetrazol-1yl)aniline $(\mathbf{4 c})$

Anal. Calcd. (\%) for $\mathrm{C}_{21} \mathrm{H}_{14} \mathrm{FN}_{5} \mathrm{O}_{2}$ : C, 65.11; H, 3.64; N, 18.08. Found (\%): C, 65.07; H, 3.62; N, 18.04; Yellow solid; m. p.145-147 ${ }^{\circ} \mathrm{C} ; \mathrm{R}_{\mathrm{f}}=0.49$; FT-IR $\left(\mathrm{KBr}, \mathrm{cm}^{-1}\right): 1603,1594$; ${ }^{1} \mathrm{H}$ NMR (300 MHz, $\left.\mathrm{CDCl}_{3}, \delta / \mathrm{ppm}\right): 7.92(\mathrm{~s}, 1 \mathrm{H}), 7.82-6.50(\mathrm{~m}, 11 \mathrm{H}), 6.01(\mathrm{~s}, 2 \mathrm{H}) ;{ }^{13} \mathrm{C}$ NMR (75 MHz, DMSO- $\left.d_{6}, \delta / \mathrm{ppm}\right): 165.05,152.83,151.51,148.99,147.67,146.55,136.89$, $135.44,131.61,129.20,128.74,128.07,126.65,121.73,120.83,109.90,106.52,102.07$; LC-MS $(\mathrm{m} / z) 387.1209$.

(E)-N-(benzo[d][1,3]dioxol-5-ylmethylene)-2-(5-(4-methoxyphenyl)-1H-tetrazol-1yl)aniline $(\mathbf{4 d})$

Anal. Calcd. (\%) for $\mathrm{C}_{22} \mathrm{H}_{17} \mathrm{~N}_{5} \mathrm{O}_{3}$ : C, 66.16; H, 4.29; N, 17.53. Found (\%):C, 66.14; H, 4.25; N, 17.50; Brown solid; m. p.112-14 ${ }^{\circ} \mathrm{C} ; \mathrm{R}_{\mathrm{f}}=0.39$; FT-IR $\left(\mathrm{KBr}, \mathrm{cm}^{-1}\right): 1607,1581 ;{ }^{1} \mathrm{H}$ NMR $(300$ $\left.\mathrm{MHz}, \mathrm{CDCl}_{3}, \delta / \mathrm{ppm}\right): \quad 9.43(\mathrm{~s}, 1 \mathrm{H}), 7.98-6.52(\mathrm{~m}, 11 \mathrm{H}), 5.98(\mathrm{~s}, 2 \mathrm{H}), 3.85(\mathrm{~s}, 3 \mathrm{H}) ;{ }^{13} \mathrm{C}$ $\operatorname{NMR}\left(75 \mathrm{MHz}, \mathrm{DMSO}-d_{6}, \delta / \mathrm{ppm}\right): 165.34,155.83,149.03,147.91,146.11,137.29,135.08$, 
131.66, 130.87, 128.61, 127.52, 125.68, 121.48, 120.66, 109.11, 106.57, 101.99, 54.36; LC$\operatorname{MS}(\mathrm{m} / \mathrm{z}): 399.1201$.

(E)-N-(benzo[d][1,3]dioxol-5-ylmethylene)-2-(5-(p-tolyl)-1H-tetrazol-1-yl)aniline (4e) Anal. Calcd. (\%) forC $\mathrm{C}_{22} \mathrm{H}_{17} \mathrm{~N}_{5} \mathrm{O}_{2}$ : C, 68.92; H, 4.47; N, 18.27; Found (\%): C, 68.88; H, 4.43; N, 18.25; Yellow solid; m. p. $137-139{ }^{\circ} \mathrm{C} ; \mathrm{R}_{\mathrm{f}}=0.40$; FT-IR $\left(\mathrm{KBr}, \mathrm{cm}^{-1}\right): 1609,1573 ;{ }^{1} \mathrm{H}$ NMR $(300$ $\left.\mathrm{MHz}, \mathrm{CDCl}_{3}, \delta / \mathrm{ppm}\right): 9.23(\mathrm{~s}, 1 \mathrm{H}), 7.80-6.55(\mathrm{~m}, 11 \mathrm{H}), 6.02(\mathrm{~s}, 2 \mathrm{H}), 2.40(\mathrm{~s}, 3 \mathrm{H}) ;{ }^{13} \mathrm{C} \mathrm{NMR}(75$ MHz, DMSO- $\left.d_{6}, \delta / \mathrm{ppm}\right): 165.28,158.50,150.01,147.96,146.72,137.20,134.91,131.50,130.97$, 128.34, 127.55, 125.52, 121.29, 120.61, 108.37, 106.10, 101.64, 20.96; LC-MS (m/z) 383.3875.

(E)-N-(benzo[d][1,3]dioxol-5-ylmethylene)-4-(5-phenyl-1H-tetrazol-1-yl)aniline (4f)

Anal. Calcd. (\%) for $\mathrm{C}_{21} \mathrm{H}_{15} \mathrm{~N}_{5} \mathrm{O}_{2}: \mathrm{C}, 68.28 ; \mathrm{H}, 4.09 ; \mathrm{N}, 18.96$. Found (\%): C, 68.27; H, 4.07; N, 18.95; Colorless solid; m. p.155- $157{ }^{\circ} \mathrm{C} ; \mathrm{R}_{\mathrm{f}}=0.43$; FT-IR $\left(\mathrm{KBr}, \mathrm{cm}^{-1}\right): 1604,1578 ;{ }^{1} \mathrm{H}$ NMR (300 MHz, $\left.\mathrm{CDCl}_{3}, \delta / \mathrm{ppm}\right): 8.37(\mathrm{~s}, 1 \mathrm{H}), 7.71-6.52(\mathrm{~m}, 12 \mathrm{H}), 6.04(\mathrm{~s}, 2 \mathrm{H}) ;{ }^{13} \mathrm{C} \mathrm{NMR}(75 \mathrm{MHz}$, DMSO- $\left.d_{6}, \delta / \mathrm{ppm}\right): 165.41,158.33,151.11,148.19,147.02,137.38,134.27,130.89,129.92$, 128.51, 128.01, 126.49, 121.77, 120.61, 107.84, 106.33, 101.71; LC-MS (m/z): 369.1236.

(E)-N-(benzo[d][1,3]dioxol-5-ylmethylene)-4-(5-(4-chlorophenyl)-1H-tetrazol-1yl)aniline $\mathbf{( 4 g )}$

Anal. Calcd.(\%) for $\mathrm{C}_{21} \mathrm{H}_{14} \mathrm{ClN}_{5} \mathrm{O}_{2}$ : C, 62.46; H, 3.49; N, 17.34. Found (\%): C, 62.43; H, 3.47 ; N, 17.32; Brown solid; m. p.144-146 ${ }^{\circ} \mathrm{C} ; \mathrm{R}_{\mathrm{f}}=0.44$; FT-IR $\left(\mathrm{KBr}, \mathrm{cm}^{-1}\right): 1622,1595 ;{ }^{1} \mathrm{H}$ NMR $(300$ $\left.\mathrm{MHz}, \mathrm{CDCl}_{3}, \delta / \mathrm{ppm}\right): 8.38(\mathrm{~s}, 1 \mathrm{H}), 7.84-6.48(\mathrm{~m}, 11 \mathrm{H}), 6.04(\mathrm{~s}, 2 \mathrm{H}) ;{ }^{13} \mathrm{C}$ NMR $(75 \mathrm{MHz}$, DMSO$\left.d_{6}, \delta / \mathrm{ppm}\right): 164.26,158.96,150.11,149.01,147.96,146.87,136.97,134.83,133.58,130.95$, 129.56, 128.40, 125.67, 121.89, 120.67, 108.37, 106.16, 101.67; LC-MS (m/z): 403.3265.

(E)-N-(benzo[d][1,3]dioxol-5-ylmethylene)-4-(5-(4-fluorophenyl)-1H-tetrazol-1yl)aniline (4h)

Anal. Calcd. (\%) forC ${ }_{21} \mathrm{H}_{14} \mathrm{FN}_{5} \mathrm{O}_{2}: \mathrm{C}, 65.11 ; \mathrm{H}, 3.64 ; \mathrm{N}, 18.08$. Found (\%): C, 65.09; H, $3.63 ; \mathrm{N}$, 18.06; Colorless solid; m. p.137-139 ${ }^{\circ} \mathrm{C} ; \mathrm{R}_{\mathrm{f}}=0.51$; FT-IR $\left(\mathrm{KBr}, \mathrm{cm}^{-1}\right): 1605,1593 ;{ }^{1} \mathrm{H}$ NMR (300 MHz, $\left.\mathrm{CDCl}_{3}, \delta / \mathrm{ppm}\right): 8.36$ (s, 1H), 7.83-6.57 (m, 11H), $6.05(\mathrm{~s}, 2 \mathrm{H}) ;{ }^{13} \mathrm{C} \mathrm{NMR}(75 \mathrm{MHz}$, DMSO- $\left.d_{6}, \delta / \mathrm{ppm}\right): 165.22,164.26,162.74,158.54,150.02,147.96,146.79,137.08,134.86$, 131.34, 130.96, 125.53, 121.89, 120.99, 115.38, 108.36, 106.09, 101.65; LC-MS(m/z): 387.3789.

(E)-N-(benzo[d][1,3]dioxol-5-ylmethylene)-4-(5-(4-methoxyphenyl)-1H-tetrazol-1yl)aniline (4i)

Anal. Calcd. (\%) for $\mathrm{C}_{22} \mathrm{H}_{17} \mathrm{~N}_{5} \mathrm{O}_{3}: \mathrm{C}, 66.16 ; \mathrm{H}, 4.29 ; \mathrm{N}, 17.53$. Found (\%): C, 66.14; H, 4.27; N, 17.51; Yellow solid; m. p.150-152 ${ }^{\circ} \mathrm{C} ; \mathrm{R}_{\mathrm{f}}=0.42$; FT-IR $\left(\mathrm{KBr}, \mathrm{cm}^{-1}\right): 1609,1594 ;{ }^{1} \mathrm{H}$ NMR (300 $\left.\mathrm{MHz}, \mathrm{CDCl}_{3}, \delta / \mathrm{ppm}\right): 8.38(\mathrm{~s}, 1 \mathrm{H}), 7.86-6.89(\mathrm{~m}, 11 \mathrm{H}), 6.04(\mathrm{~s}, 2 \mathrm{H}), 3.87(\mathrm{~s}, 3 \mathrm{H}) ;{ }^{13} \mathrm{C} \mathrm{NMR}(75$ MHz, DMSO- $\left.d_{6}, \delta / \mathrm{ppm}\right): 165.31,148.33,141.52,139.36,132.03,131.46,129.29,128.85,127.70$, 126.87, 115.97, 112.98, 108.55, 106.26, 102.29, 101.56, 55.38; LC-MS (m/z): 399.4026.

(E)-N-(benzo[d][1,3]dioxol-5-ylmethylene)-4-(5-(p-tolyl)-1H-tetrazol-1-yl)aniline $(\mathbf{4 j})$ Anal. Calcd. (\%) for $\mathrm{C}_{22} \mathrm{H}_{17} \mathrm{~N}_{5} \mathrm{O}_{2}$ : C, 68.92; H, 4.47; N, 18.27. Found (\%): C, 68.90; H, 4.45; N, 18.26; Pale yellow solid;m. p. 159-161 ${ }^{\circ} \mathrm{C} ; \mathrm{R}_{\mathrm{f}}=0.46$; FT-IR $\left(\mathrm{KBr}, \mathrm{cm}^{-1}\right): 1605,1590 ;{ }^{1} \mathrm{H}$ NMR (300 MHz, $\left.\mathrm{CDCl}_{3}, \delta / \mathrm{ppm}\right): 8.37(\mathrm{~s}, 1 \mathrm{H}), 7.86-6.89(\mathrm{~m}, 11 \mathrm{H}), 6.03(\mathrm{~s}, 2 \mathrm{H}), 2.42$ (s, $3 \mathrm{H}) ;{ }^{13} \mathrm{C}$ NMR $\left(75 \mathrm{MHz}, \mathrm{DMSO}-d_{6}, \delta / \mathrm{ppm}\right): 165.19,158.94,150.11,149.01,147.96$, $146.60,141.50,137.30,134.89,132.04,128.86,127.62,125.68,121.89,120.92,108.36$, 106.16, 101.67; LC-MS(m/z): 383.4089. 
(E)-N-(benzo[d][1,3]dioxol-5-ylmethylene)-3-(5-phenyl-1H-tetrazol-1-yl)aniline (4k)

Anal. Calcd. (\%) forC $\mathrm{C}_{21} \mathrm{H}_{15} \mathrm{~N}_{5} \mathrm{O}_{2}$ : C, 68.28; H, 4.09; N, 18.96. Found (\%): C, $68.27 ; \mathrm{H}, 4.08$; N, 18.95;Colorless solid;m. p.190-192 ${ }^{\circ} \mathrm{C} ; \mathrm{R}_{\mathrm{f}}=0.46$; FT-IR $\left(\mathrm{KBr}, \mathrm{cm}^{-1}\right): 1601,1582 ;{ }^{1} \mathrm{H}$ NMR (300 MHz, $\left.\mathrm{CDCl}_{3}, \delta / \mathrm{ppm}\right): 9.81(\mathrm{~s}, 1 \mathrm{H}), 7.79-6.67(\mathrm{~m}, 12 \mathrm{H}), 5.97$ (s, 2H); ${ }^{13} \mathrm{C}$ NMR (75 MHz, DMSO- $\left.d_{6}, \delta / \mathrm{ppm}\right): 165.54,139.32,134.93,131.52,129.08,128.73,128.54$, 128.33, 127.67, 116.07, 112.95, 108.55, 106.26, 102.30, 100.83; LC-MS (m/z): 369.1311.

(E)-N-(benzo[d][1,3]dioxol-5-ylmethylene)-3-(5-(4-chlorophenyl)-1H-tetrazol-1yl)aniline (4l)

Anal. Calcd. (\%) for $\mathrm{C}_{21} \mathrm{H}_{14} \mathrm{ClN}_{5} \mathrm{O}_{2}: \mathrm{C}, 62.46 ; \mathrm{H}, 3.49 ; \mathrm{N}, 17.34$. Found (\%): C, 62.44; H, $3.47 ; \mathrm{N}$, 17.32;Pale yellow solid; m. p. $187-189{ }^{\circ} \mathrm{C} ; \mathrm{R}_{\mathrm{f}}=0.52$; FT-IR $\left(\mathrm{KBr}, \mathrm{cm}^{-1}\right): 1623,1594 ;{ }^{1} \mathrm{H}$ NMR (300 MHz, $\left.\mathrm{CDCl}_{3}, \delta / \mathrm{ppm}\right): 9.82(\mathrm{~s}, 1 \mathrm{H}), 7.72-6.45(\mathrm{~m}, 11 \mathrm{H}), 5.97(\mathrm{~s}, 2 \mathrm{H}) ;{ }^{13} \mathrm{C} \mathrm{NMR}(75 \mathrm{MHz}$, DMSO- $\left.d_{6}, \delta / \mathrm{ppm}\right): 164.48,139.17,136.41,136.15,133.61,130.99,129.66,129.56,128.95$, 128.56, 128.44, 116.23, 112.95, 108.58, 108.44, 106.29, 102.32, 101.75; LC-MS (m/z): 403.0340.

(E)-N-(benzo[d][1,3]dioxol-5-ylmethylene)-3-(5-(4-fluorophenyl)-1H-tetrazol-1yl)aniline (4m)

Anal. Calcd. (\%) for $\mathrm{C}_{21} \mathrm{H}_{14} \mathrm{FN}_{5} \mathrm{O}_{2}$ : C, 65.11; H, 3.64; N, 18.08. Found (\%): C, 65.09; H, 3.62; N, 18.06; Colorless solid; m. p.197-198 ${ }^{\circ} \mathrm{C} ; \mathrm{R}_{\mathrm{f}}=0.53$; FT-IR $\left(\mathrm{KBr}, \mathrm{cm}^{-1}\right): 1604,1589 ;{ }^{1} \mathrm{H}$ NMR (300 MHz, $\left.\mathrm{CDCl}_{3}, \delta / \mathrm{ppm}\right): 9.84(\mathrm{~s}, 1 \mathrm{H}), 7.86-6.54(\mathrm{~m}, 11 \mathrm{H}), 5.98(\mathrm{~s}, 2 \mathrm{H}) ;{ }^{13} \mathrm{C} \mathrm{NMR}(100 \mathrm{MHz}$, DMSO- $\left.d_{6}, \delta / \mathrm{ppm}\right): 165.28,164.41,162.80,139.23,131.46,131.34,131.31,130.45,130.36$, $128.57,116.09,115.37,115.15,112.89,108.55,106.26,102.29$; LC-MS $(m / z): 387.3741$.

(E)-N-(benzo[d][1,3]dioxol-5-ylmethylene)-3-(5-(4-methoxyphenyl)-1H-tetrazol-1yl)aniline (4n)

Anal. Calcd. (\%) for $\mathrm{C}_{22} \mathrm{H}_{17} \mathrm{~N}_{5} \mathrm{O}_{3}: \mathrm{C}, 66.16 ; \mathrm{H}, 4.29 ; \mathrm{N}, 17.53$. Found (\%):C, 66.14; H, 4.27; N, 17.51;Colorless solid; m. p. $182-183{ }^{\circ} \mathrm{C} ; \mathrm{R}_{\mathrm{f}}=0.58$; FT-IR $\left(\mathrm{KBr}, \mathrm{cm}^{-1}\right): 1605,1557 ;{ }^{1} \mathrm{H}$ NMR (300 MHz, $\left.\mathrm{CDCl}_{3}, \delta / \mathrm{ppm}\right): 9.80(\mathrm{~s}, 1 \mathrm{H}), 7.99-6.07(\mathrm{~m}, 11 \mathrm{H}), 5.96(\mathrm{~s}, 2 \mathrm{H}), 3.87(\mathrm{~s}$, $3 \mathrm{H}) ;{ }^{13} \mathrm{C}$ NMR $\left(75 \mathrm{MHz}, \mathrm{DMSO}-d_{6}, \delta / \mathrm{ppm}\right): 165.89,164.84,163.10,161.86,139.43,131.19$, 129.60, 126.93, 121.80, 115.85, 114.00, 113.54, 112.95, 51.77; LC-MS (m/z): 399.4012.

(E)-N-(benzo[d][1,3]dioxol-5-ylmethylene)-3-(5-(p-tolyl)-1H-tetrazol-1-yl)aniline (4o)

Anal. Calcd. (\%) for $\mathrm{C}_{22} \mathrm{H}_{17} \mathrm{~N}_{5} \mathrm{O}_{2}$ : C, 68.92; H, 4.47; N, 18.27. Found (\%): C, 68.90; H, 4.45; N, 18.24; Colorless solid; m. p. $175-176{ }^{\circ} \mathrm{C} ; \mathrm{R}_{\mathrm{f}}=0.59$; FT-IR $\left(\mathrm{KBr}, \mathrm{cm}^{-1}\right): 1607,1591 ;{ }^{1} \mathrm{H}$ NMR $\left(300 \mathrm{MHz}, \mathrm{CDCl}_{3}, \delta / \mathrm{ppm}\right): 9.79(\mathrm{~s}, 1 \mathrm{H}), 7.57-6.26(\mathrm{~m}, 11 \mathrm{H}), 5.97(\mathrm{~s}, 2 \mathrm{H}), 2.58$ $(\mathrm{s}, 3 \mathrm{H}) ;{ }^{13} \mathrm{C}$ NMR $\left(75 \mathrm{MHz}, \mathrm{DMSO}-d_{6}, \delta / \mathrm{ppm}\right): 165.42,148.51,142.56,139.74,132.51$, 131.23, 129.62, 128.99, 128.05, 126.63, 116.09, 112.74, 108.38, 106.85, 102.59, 101.35, 21.55; LC-MS $(m / z): 383.4019$.

\section{Results and Discussion}

We started our investigation by employing piperonal, o-phenyl diamine with benzoyl chloride in the presence of $\mathrm{PCl}_{5}$ and $\mathrm{NaN}_{3}$ as model substrates. As we expected the reaction was successful and gave the desired biaryl tetrazole. However, the yield of expected tetrazole is very low. Hence, the reaction was optimized to increase the yield and find out the most suitable conditions for the synthesis of biaryl tetrazole. We studied the various parameters such as solvents, base and temperature to achieve suitable conditions and the results are summarized in Table 1-3. 
It was observed that, among all solvents, the best result (80\%) was obtained in ethanol (Table 1). Further, the increasing volume of solvent did not affect the yield of tetrazole. Next, variety of organic and inorganic bases were utilized this reaction. Inorganic bases such as $\mathrm{KOH}, \mathrm{NaOH}, \mathrm{K}_{2} \mathrm{CO}_{3}, \mathrm{Na}_{2} \mathrm{CO}_{3}$, were effectively increases the target product and found $\mathrm{KOH}$ is the best one. In spite of, organic bases such as pyridine, piperidine and triethylamine were did not increase the yield of expected product (Table 2). The expected product was not obtained when the reaction was carried out in the absence of $\mathrm{PCl}_{5}$. Thus prove the crucial role of $\mathrm{PCl}_{5}$ in the conversion of secondary amide to tetrazole in situ.

Table 1. Effect of solvents

\begin{tabular}{ccc}
\hline Entry & Solvent & Yield, \% \\
\hline 1 & Ethanol & 78 \\
2 & Methanol & 72 \\
3 & $n$-Propanol & 68 \\
4 & Toluene & 61 \\
5 & DMF & 57 \\
6 & DMAc & 59 \\
7 & DMSO & 52 \\
\hline
\end{tabular}

Table 2. Effect of bases

\begin{tabular}{ccc}
\hline Entry & Base & Yield, $\%$ \\
\hline 1 & No base & 35 \\
2 & $\mathrm{KOH}$ & 78 \\
3 & $\mathrm{~K}_{2} \mathrm{CO}_{3}$ & 71 \\
4 & $\mathrm{NaOH}$ & 74 \\
5 & $\mathrm{Na}_{2} \mathrm{CO}_{3}$ & 65 \\
6 & Pyridine & 40 \\
7 & Piperidine & 35 \\
8 & Triethylamine & 49 \\
\hline
\end{tabular}

Table 3. Effect of temperature

\begin{tabular}{ccc}
\hline Entry & Temperature, ${ }^{\circ} \mathrm{C}$ & Yield, $\%$ \\
\hline 1 & RT & No reaction \\
2 & 40 & 55 \\
3 & 50 & 68 \\
4 & 60 & 79 \\
5 & 70 & 88 \\
6 & 80 & 87 \\
7 & 90 & 71 \\
\hline
\end{tabular}

We further examined the effect of temperature on the tetrazole synthesis. At the beginning, the reaction was carried out in room-temperature. No progress was observed and hence the same reaction was studied by increasing the temperature from $50-90{ }^{\circ} \mathrm{C}$. Higher yield was obtained at $70{ }^{\circ} \mathrm{C}$. No significant increase in the yield of product was observed as the reaction temperature was raised from $70{ }^{\circ} \mathrm{C}$ to $110{ }^{\circ} \mathrm{C}$. Therefore, $70{ }^{\circ} \mathrm{C}$ was chosen for this reaction. With this optimized reaction conditions in hand we next explore the reaction scope. A variety of aryl diamine and benzoylchlorides possessing either electron-donating or -withdrawing groups.

Generally, all the aryl diamines (2a-2c) and benzoyl chlorides (3a-3e) underwent above resulting protocol smoothly and afforded expected products in good yields. In all the cases, the reactions proceeded in an excellent regioselective manner and provided only a particular regioisomer as sole product. At the outset, sterically hindered ortho-phenydiamine was allow to react with piperonal and substituted benzoyl chlorides (3a-3e) underwent this reaction successfully and provided $\mathbf{4 a - 4 e}$ in $80-88 \%$ of isolated yields. Among those, unsubstituted benzoyl chloride gave $88 \%$ of the desired product (Scheme 1).

Next, the reaction of 1a with para-phenyl diamine (2b) and benzoyl chlorides (3a-3e) gave $\mathbf{4 f - 4 j}$ in an excellent yield (87-89\%). $89 \%$ of the tiled product was obtained by using unsubstituted benzoyl chloride. In the same way, the reaction of meta-phenyl diamine (2c) with 1a and 3a-3e smoothly participated and provided biaryl tetrazoles (4k-4o) in good quantities. The current investigation has merits over the existing methods in metal catalysts 
were employed. Facile manner of synthesis, use of simple reagents, ease of isolation of products and milder reaction conditions are the unique features of this method.

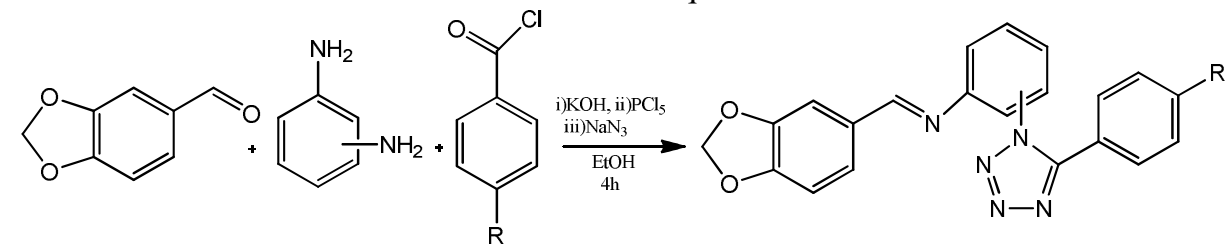

1a

2a-2c

3a-3e

4a-4o<smiles>Fc1ccc(-c2nnnn2-c2ccccc2N=Cc2ccc3c(c2)OCO3)cc1</smiles>

4a, $88 \%$

4b, $85 \%$

4c, $83 \%$<smiles>COc1ccc(-c2nnnn2-c2ccccc2N=Cc2ccc3c(c2)OCO3)cc1</smiles>

4d, $80 \%$<smiles>Clc1ccc(-c2nnnn2-c2ccc(N=Cc3ccc4c(c3)OCO4)cc2)cc1</smiles><smiles>Fc1ccc(-c2nnnn2-c2ccc(N=Cc3ccc4c(c3)OCO4)cc2)cc1</smiles>

4h, $87 \%$<smiles>COc1ccc(-c2nnnn2-c2ccc(N=Cc3ccc4c(c3)OCO4)cc2)cc1</smiles>

$4 \mathbf{i}, 88 \%$<smiles>Cc1ccc(-c2nnnn2-c2ccc(/N=C/c3ccc4c(c3)OCO4)cc2)cc1</smiles><smiles>C(=N/c1cccc(-n2nnnc2-c2ccccc2)c1)\c1ccc2c(c1)OCO2</smiles>

$4 \mathrm{k}, 82 \%$<smiles>Clc1ccc(-c2nnnn2-c2cccc(/N=C/c3ccc4c(c3)OCO4)c2)cc1</smiles><smiles>Fc1ccc(-c2nnnn2-c2cccc(/N=C/c3ccc4c(c3)OCO4)c2)cc1</smiles><smiles>COc1ccc(-c2nnnn2-c2cccc(/N=C/c3ccc4c(c3)OCO4)c2)cc1</smiles>

4n, $70 \%$<smiles>Cc1ccc(-c2nnnn2-c2cccc(/N=C/c3ccc4c(c3)OCO4)c2)cc1</smiles>

4o, $82 \%$

Scheme 1. Syntheses of tetrazole derivatives

\section{Reaction mechanism}

The reaction mechanism (Scheme 2) involves three steps. The step 1 involved the condensation reaction of piperanol with ortho-phenylene diamine to form Schiff base. First, the amine nitrogen acts as a nucleophile, attacking the carbonyl carbon. This is closely analogous to 
hemiacetal and hemiketal formation. In the next step, Schotten-Baumann reaction of Schiff base derived from step 1 with benzoyl chloride provided secondary amide. In the final step, secondary amide initially reacts with $\mathrm{PCl}_{5}$ and gives an intermediate. This intermediate further reacted with $\mathrm{NaN}_{3}$ to yield expected tetrazole through cyclization process.

Step 1:

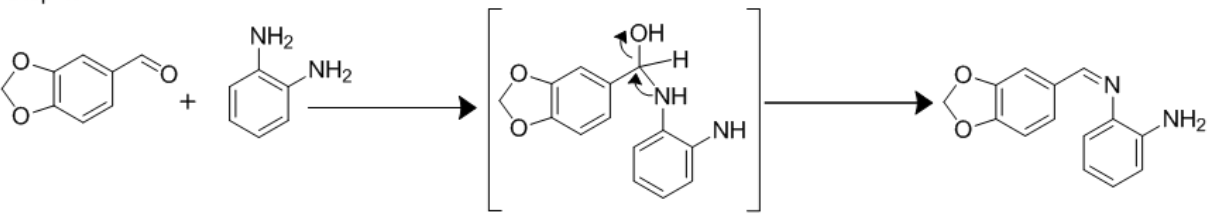

Step 2:

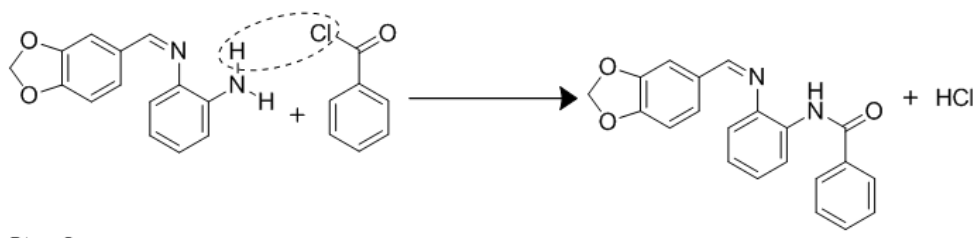

Step 3:

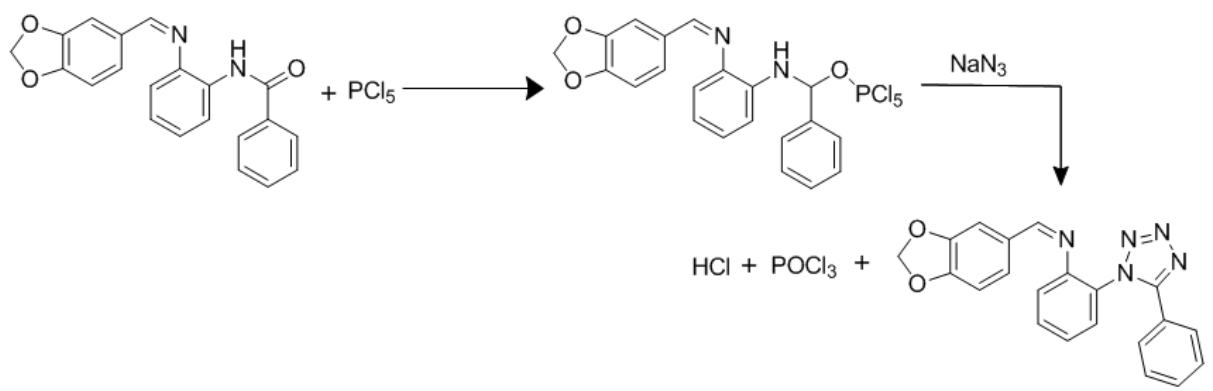

Scheme 2. Proposed reaction mechanism of the synthesis of tetrazoles from secondary amide in situ

\section{Conclusion}

We have developed an extremely efficient and metal free protocol for the synthesis of biologically active tetrazole derivatives. All the synthesized products were confirmed through IR, ${ }^{1} \mathrm{H},{ }^{13} \mathrm{C}$ NMR and LC-MS. This is the first report of the synthesis of tetrazoles via multicomponent reaction of piperonal, various aryldiamines, substituted benzoylchlorides, in the presence of $\mathrm{PCl}_{5}$ and $\mathrm{NaN}_{3}$ an inexpensive azide source. The advantages of this new method are operational simplicity; high to excellent yields of products in short reaction times and easy workup procedures.

\section{References}

1. Roh J, Vávrová K and Hrabálek A, Eur J Org Chem., 2012, 2012(31), 6101-6118; DOI:10.1002/ejoc.201200469

2. Yue T, Wang M X, Wang D X and Zhu J, Angew Chem Int Ed., 2008, 47(49), 9454-9457; DOI:10.1002/anie.200804213

3. Patani G and LaVoie E J, Chem Rev., 1996, 96(8), 3147-3176; DOI:10.1021/cr950066q 
4. Singh H, Chawla A S, Kapoor V K, Paul D and Malhotra P K, Prog Med Chem., 1980, 17, 151-183; DOI:10.1016/S0079-6468(08)70159-0

5. McManus J M and Herbst R M, J Org Chem., 1959, 24(11), 1643-1649; DOI:10.1021/jo01093a006

6. Herr R J, Bioorg Med Chem., 2002, 10(11), 3379-3393; DOI:10.1016/S09680896(02)00239-0

7. Myznikov L V, Hrabalek A and Koldobskii G I, Chem Heterocycl Compd., 2007, 43(1), 1-9; DOI:10.1007/s10593-007-0001-5

8. Gutmann B, Roduit J P, Roberge D and Kappe C O, Angew Chem Int Ed., 2010, 49(39), 7101-7105; DOI:10.1002/anie.201003733

9. Mohite P B and Bhaskar V H, Int J Pharm Tech Res., 2011, 3(3), 1557-1566.

10. Osheroff N, Zechiedrich E L and Gale K C, BioEssays, 1991, 13(6), 269-275; DOI:10.1002/bies.950130603

11. Issell B F, Cancer Chemother Pharmacol., 1982, 7(2-3), 73-80.

12. Arshad M, Bhat A R, Pokharel S, Kim J E, Lee E J, Athar F and Choi I, Eur J Med Chem., 2014,71, 229-236; DOI:10.1016/j.ejmech.2013.11.008

13. Marquez Alvarez H, Barbosa D P, Fricks A T, Aranda D A G, Valdés R H and Antunes O A C, Org Proc Res Dev., 2006, 10, 941-943; DOI:10.1021/op060117t

14. Cortés-Salazar F, Avella-Moreno E, Cortés M T and Fidel Suárez-Herrera M, J Electroanal Chem., 2007, 606(1), 1-7; DOI:10.1016/j.jelechem.2007.04.003

15. Aukunuru J, Eedula K, Pasham V, Katla V and Reddy S K, Int J Pharm Sci Nanotechnol., 2009, 2(1), 435-442.

16. Bjørsvik H R, Liguori L and Minisci F, Org Proc Res Dev., 2000, 4(6), 534-543; DOI:10.1021/op0000529

17. Bellardita M, Loddo V, Palmisano G, Pibiri I, Palmisano L and Augugliaroaa V, Appl Catal B Environ., 2014, 144, 607-613; DOI:10.1016/j.apcatb.2013.07.070

18. de Oliveira A N, Bocca C C, Carvalho J E, Ruiz A L G, Silva T P, Rittner R and Hoehr N F, Eur J Med Chem., 2010, 45(9), 4339-4342; DOI:10.1016/j.ejmech.2010.04.034

19. Mathew A, Mary Sheeja T L, Kumar A T and Radha K, Hyg J Dug Med., 2011, 3, 48-56.

20. Prasanthi G, Prasad K V and Bharathi K, Eur J Med Chem., 2013, 66, 516-525; DOI:10.1016/j.ejmech.2013.06.006

21. Aboul-Enein M N, El-Azzouny A A, Attia M I, Maklad Y A, Amin K M, AbdelRehim M and El-Behairy M F, Eur J Med Chem., 2012, 47(1), 360-369; DOI:10.1016/j.ejmech.2011.11.004

22. Wani M Y, Bhat A R, Azam A, Choi I and Fareeda Athar, Eur J Med Chem. ,2012, 48, 313-320; DOI:10.1016/j.ejmech.2011.12.033

23. Alizadeh B H, Foroumadi A, Emami S, Khoobi M, Panah F, Ardestani S K and Shafiee A, Eur J Med Chem., 2010, 45(12), 5979-5984;

DOI:10.1016/j.ejmech.2010.09.064

24. Yeo H, Li Y, Fu L, Zhu J L, Gullen E A, Dutschman G E, Lee Y, Chung R, Huang E S, Austin D J and Cheng Y C, J Med Chem., 2005, 48(2), 534-546; DOI:10.1021/jm034265a

25. Feng W, Satyanarayana M, Tsai Y C, Liu A A, Liu L F and LaVoie E J, Eur J Med Chem., 2009, 44(9), 3433-3438; DOI:10.1016/j.ejmech.2009.02.004

26. Beghyn T B, Charton J, Leroux F, Henninot A, Reboule I, Cos P, Maes L and Deprez B, J Med Chem., 2012, 55(3), 1274-1286; DOI:10.1021/jm201422e

27. Khanapure S P, Garvey D S, Young D V, Ezawa M, Earl R A, Gaston R D, Fang X, Murty M, Martino A, Shumway M, Trocha M, Marek P, Tam S W, Janero D R and Letts L G, J Med Chem., 2003, 46(25), 5484-5504; DOI:10.1021/jm030268b 